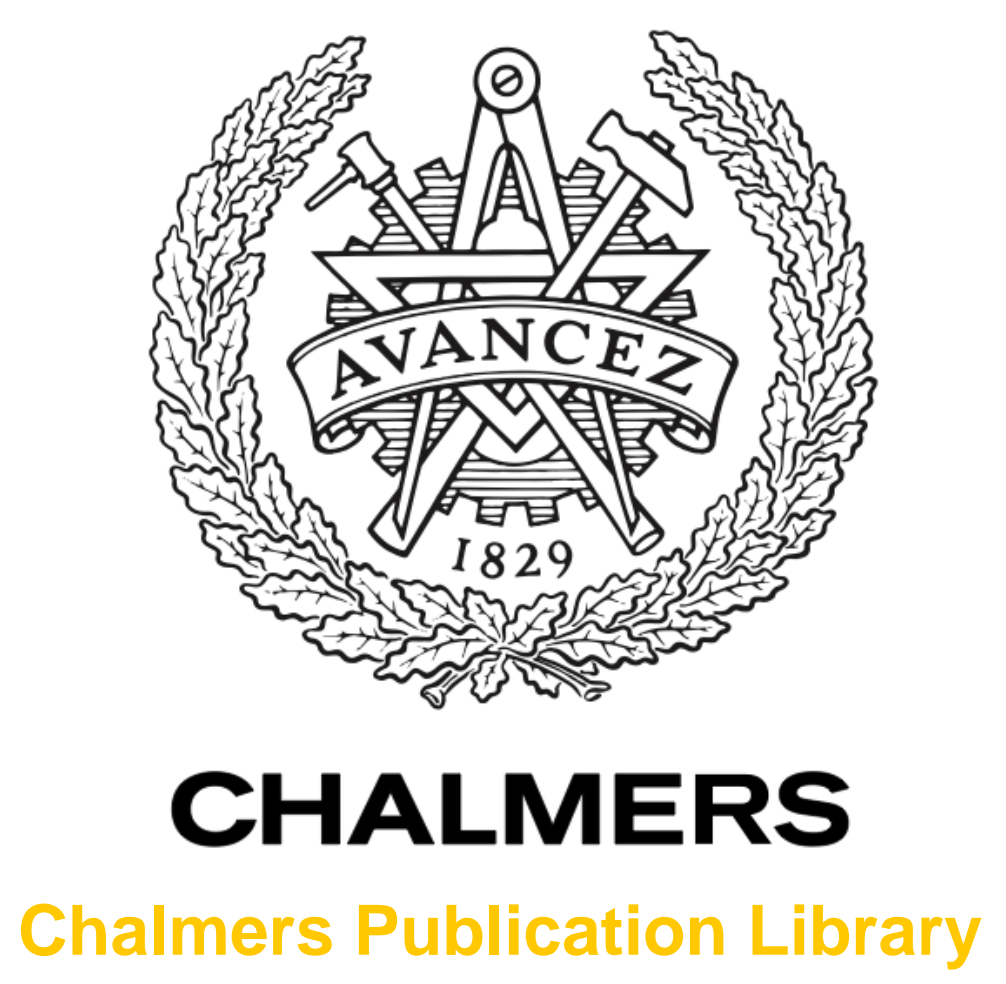

On the sum rate of MIMO Nakagami-m fading channels with MMSE receivers

This document has been downloaded from Chalmers Publication Library $(\mathrm{CPL})$. It is the author's version of a work that was accepted for publication in:

Proceedings of the IEEE Sensor Array and Multichannel Signal Processing Workshop (ISSN: 2151-870X)

Citation for the published paper:

Zhong, C. ; Matthaiou, M. ; Huang, A. (2012) "On the sum rate of MIMO Nakagami-m fading channels with MMSE receivers". Proceedings of the IEEE Sensor Array and

Multichannel Signal Processing Workshop pp. 49-52.

http://dx.doi.org/10.1109/SAM.2012.6250549

Downloaded from: http://publications.lib.chalmers.se/publication/164996

Notice: Changes introduced as a result of publishing processes such as copy-editing and formatting may not be reflected in this document. For a definitive version of this work, please refer to the published source. Please note that access to the published version might require a subscription. 


\title{
On the Sum Rate of MIMO Nakagami- $m$ Fading Channels with MMSE Receivers
}

\author{
Caijun Zhong ${ }^{\S}$, Michail Matthaiou ${ }^{\dagger}$, Aiping Huang ${ }^{\S}$, and Zhaoyang Zhang ${ }^{\S}$ \\ $\S$ Institute of Information and Communication Engineering, Zhejiang University, Hangzhou, China \\ ${ }^{\dagger}$ Department of Signals and Systems, Chalmers University of Technology, Gothenburg, Sweden \\ Email: \{caijunzhong, aiping.huang, ning_ming\}@zju.edu.cn, michail.matthaiou@chalmers.se
}

\begin{abstract}
In this paper, we elaborate on the ergodic sum rate of multiple-input multiple-output (MIMO) Nakagami- $m$ fading channels with linear minimum mean square error (MMSE) receivers. For dual transmit antenna configurations, we present new, closed-form upper bounds on the ergodic sum rate of MMSE receivers. Moreover, we derive exact expressions for the two key parameters dictating the sum rate behavior in the low signal to noise ratio (SNR) regime, i.e., minimum energy per information bit to reliably convey any positive rate and the wideband slope. Our analytical results demonstrate the sub-optimality of linear MMSE receivers and provide useful insights into the impact of model parameters (e.g. number of antennas, fading parameters).
\end{abstract}

\section{INTRODUCTION}

The seminal discovery of [1], which suggested that deploying multiple antennas at both the transmitter and receiver can significantly increase the spectral efficiency of communication systems, has triggered enormous interest in understanding the capacity limits of MIMO systems in various practical propagation environments. In particular, the effects of antenna correlation, line-of-sight, frequency selectivity and keyhole phenomenon on the capacity of MIMO systems have been well studied among others (see for example [2]-[4] and references therein). The common characteristic of the above mentioned works is that they all assume the use of optimal nonlinear maximum likelihood (ML) receivers. While ML receivers achieve optimal performance, they also incur the highest implementation complexity. For this reason, low complexity linear receivers, such as minimum mean square error (MMSE) receivers, have also received a considerable amount of interest. In this context, [5] proposed a generic analytic framework for analyzing the ergodic sum rate of MIMO Rayleigh and Rician fading channels with MMSE receivers.

At the same time, it is well-known that the Nakagami-m distribution represents a more general fading model, which encompasses Rayleigh fading as a special case and can accurately approximate the Rician fading model [6]. Despite its practical importance, there have been no works studying the ergodic sum rate of MIMO Nakagami- $m$ fading channels with linear MMSE receivers, due to the difficulty in analytically evaluating the MIMO eigenstatistics. Motivated by this, we hereafter study the ergodic sum rate of MIMO Nakagami- $m$ fading channels with MMSE receivers.

In the following, we derive new, closed-form upper bounds on the ergodic sum rate of MIMO Nakagami- $m$ fading chan- nels with two transmit antennas and $N_{r} \geq 2$ receive antennas for MMSE receivers. In the low SNR regime, we obtain tractable closed-form expressions for the two key performance measures dictating the ergodic sum rate of the system, i.e., the minimum normalized energy per information bit required to convey any positive rate reliably and the wideband slope. We then analyze the impact of the fading severity parameter $m$ on the ergodic sum rate of the system, and provide a sum rate performance comparison between linear MMSE receivers and optimal ML receivers. It is worth mentioning that all the presented expressions can be very easily evaluated and, more importantly, yield useful physical insights into the factors that affect the sum rate performance of linear MMSE receivers.

The remaining of the paper is organized as follows: Section II introduces the MIMO system model. Section III examines the ergodic sum rate of MMSE receivers. Section V concludes the paper and summarizes the key findings.

Notation: We use upper and lower case boldface to denote matrices and vectors. The expectation of a random variable $(\mathrm{RV})$ is given by $\mathrm{E}\{\cdot\}$. The symbols $(\cdot)^{\dagger}$ represents the Hermitian transpose of a matrix, while the matrix determinant is denoted by $\operatorname{det}(\cdot)$, and the matrix inverse will be denoted by $(\cdot)^{-1}$. Finally, $\operatorname{tr}(\cdot)$ stands for the trace of a matrix. The symbol $\stackrel{\text { a.s. }}{\rightarrow}$ denotes almost sure convergence.

\section{MiMO System Model AND Linear Receivers}

We consider a MIMO system with $N_{t}$ transmit and $N_{r} \geq$ $N_{t}$ receive antennas. Assuming no channel state information at the transmitter, the available average power, $P$, is distributed uniformly amongst all data streams. Hence, the input-output relationship is given by

$$
\mathbf{y}=\sqrt{\frac{P}{N_{t}}} \mathbf{H s}+\mathbf{n}
$$

where $\mathbf{y} \in \mathbb{C}^{N_{r} \times 1}$ is the received signal vector, $\mathbf{s} \in \mathbb{C}^{N_{t} \times 1}$ is the vector containing the transmitted symbols which are drawn from a unit-power constellation, while the complex zero-mean noise has covariance $\mathrm{E}\left[\mathbf{n n}^{H}\right]=N_{0} \mathbf{I}_{N_{r}}$, where $N_{0}$ is the noise power. The entries of the MIMO channel matrix $\mathbf{H} \in$ $\mathbb{C}^{N_{r} \times N_{t}}$ are independent and identically distributed random variables with uniformly distributed phase in $[0,2 \pi)$, while their amplitudes $r=\left|h_{i, j}\right|$ follows a Nakagami- $m$ distribution

$$
p(r)=\frac{2}{\Gamma(m)}\left(\frac{m}{\Omega}\right)^{m} r^{2 m-1} e^{-(m / \Omega) r^{2}}, r \geq 0, m \geq \frac{1}{2},
$$


where $\Gamma(\cdot)$ is the well-known Gamma function [7, Eq. (8.310.1)] and $\Omega=\mathrm{E}\left\{r^{2}\right\}$ is the average power. Then, the squared Nakagami- $m$ envelope is gamma distributed as $r^{2} \sim \operatorname{Gamma}(m, \Omega / m)$.

As was previously mentioned, in this paper we are particularly interested in the performance analysis of linear MMSE receivers. Omitting explicit details, the post-processing SNR at the $i$-th receiver output $\left(i=1, \ldots, N_{t}\right)$ is given by [8]

$$
\gamma_{i}^{\text {mmse }} \triangleq \frac{1}{\left[\left(\mathbf{I}_{N_{t}}+\frac{\rho}{N_{t}} \mathbf{H}^{\dagger} \mathbf{H}\right)^{-1}\right]_{i, i}}-1
$$

where $\rho=P / N_{0}$ is the average SNR and $[\cdot]_{i, i}$ returns the $i$ th diagonal element of a matrix. Then, assuming independent decoding at the receiver, the achievable ergodic sum rate for both receivers can be expressed as

$$
\mathcal{R}^{\text {mmse }}(\rho) \triangleq \sum_{i=1}^{N_{t}} \mathrm{E}_{\gamma_{i}^{\text {mmse }}}\left\{\log _{2}\left(1+\gamma_{i}^{\text {mmse }}\right)\right\} .
$$

We note that, in general, a direct evaluation of (4) is difficult due to the lack of analytical closed-form expressions for the probability density function (p.d.f.) of $\gamma_{i}^{\mathrm{mmse}}$.

\section{MMSE RECEIVERS}

In this section, we study the ergodic sum rate of the MIMO Nakagami- $m$ fading channels with MMSE receivers. We first present an upper bound for the ergodic sum rate of $N_{r} \times 2$ systems at arbitrary SNRs and then look into the low SNR regime where we examine the sum rate performance of arbitrary $N_{r} \times N_{t}$ systems.

\section{A. Sum Rate Upper Bounds}

In general, an exact analysis of the ergodic sum rate of MIMO Nakagami- $m$ fading channels with MMSE receivers for arbitrary number of antennas is very difficult. Hence, we focus on the practically important case of dual-antenna systems, i.e., $N_{r} \times 2$ MIMO Nakagami- $m$ fading channels, with $N_{r} \geq 2$. Please note that these configurations are likely to be used in many future practical systems (e.g. hand-held devices), thanks to their small size and low implementation cost. We can now present the following key result:

Proposition 1: The ergodic sum rate of $N_{r} \times 2$ MIMO Nakagami- $m$ fading channels with MMSE receivers is upper bounded by

$$
\begin{aligned}
\mathcal{R}_{u}^{\mathrm{mmse}}(\rho) & =2 \log _{2}\left(\sum_{k=0}^{2}\left(\frac{\rho \Omega}{2}\right)^{k} k !\left(\begin{array}{l}
2 \\
k
\end{array}\right)\left(\begin{array}{c}
N_{r} \\
k
\end{array}\right)\right) \\
& -\frac{\rho N_{r} \Omega}{\ln 2}{ }_{3} F_{1}\left(1+m N_{r}, 1,1 ; 2 ;-\frac{\rho \Omega}{2 m}\right)
\end{aligned}
$$

where ${ }_{p} F_{q}(\cdot)$ is the generalized hypergeometric function with $p, q$ non-negative integers [7, Eq. (9.14.1)].

Proof: Due to space constraints, the proof is relegated in an extended journal version of this paper [9].

Note that Proposition 1 applies for arbitrary fading parameter $m$. For the special case of $m N_{r}$ being an integer,
Proposition 1 reduces to

$$
\begin{aligned}
\mathcal{R}_{u}^{\text {mmse }}(\rho) & =2 \log _{2}\left(\sum_{k=0}^{2}\left(\frac{\rho \Omega}{2}\right)^{k} k !\left(\begin{array}{l}
2 \\
k
\end{array}\right)\left(\begin{array}{c}
N_{r} \\
k
\end{array}\right)\right) \\
& -\frac{2}{\ln 2} \exp \left(\frac{2 m}{\rho \Omega}\right) \sum_{k=1}^{m N_{r}} E_{k}\left(\frac{2 m}{\rho \Omega}\right)
\end{aligned}
$$

where $E_{n}(x)=\int_{1}^{\infty} \exp (-x t) t^{-n} d t$ is the exponential integral function of order $n$, for $n=0,1,2, \ldots$, and $\operatorname{Re}(x)>0$.

Moreover, it can be easily shown that due to the law of large numbers, which states that $\frac{1}{N_{r}} \mathbf{H}^{\dagger} \mathbf{H} \stackrel{a . s .}{\rightarrow} \Omega \mathbf{I}_{N_{t}}$, the upper bound becomes exact for large $N_{r}$ and fixed $N_{t}$ :

$$
\mathcal{R}^{\text {mmse }}(\rho)=\mathcal{R}_{u}^{\text {mmse }}(\rho)=N_{t} \log _{2}\left(\frac{\rho N_{r} \Omega}{N_{t}}\right)+\mathcal{O}\left(\frac{1}{N_{r}}\right) .
$$

The above result indicates that increasing $N_{r}$ provides a logarithmic sum rate gain. This result is consistent with those reported in [10].

Corollary 1: In the high SNR regime, the sum rate upper bound reduces to

$$
\mathcal{R}_{u}^{\text {mmse }}(\rho) \approx 2 \log _{2}\left(\frac{\rho}{2} m \Omega N_{r}\left(N_{r}-1\right)\right)-\frac{2}{\ln 2} \psi\left(N_{r} m\right)
$$

where $\psi(\cdot)$ is the Euler's digamma function [7, Eq. (8.360.1)].

Proof: In the high SNR regime, the second term in (19) can be approximated by

$$
\begin{aligned}
& \operatorname{term}_{2}=2 \mathrm{E}_{\mathbf{h}_{i}}\left\{\log _{2}\left(\frac{\rho}{2} \mathbf{h}_{i}^{\dagger} \mathbf{h}_{i}\right)\right\} \\
& =2 \log _{2} \frac{\rho}{2}+\frac{2\left(\frac{m}{\Omega}\right)^{N_{r} m}}{\Gamma\left(N_{r} m\right) \ln 2} \int_{0}^{\infty} x^{N_{r} m-1} e^{-\frac{m x}{\Omega}} \ln x d x \\
& =2 \log _{2} \frac{\rho}{2}+\frac{2}{\ln 2}\left(\psi\left(N_{r} m\right)-\ln \frac{m}{\Omega}\right)
\end{aligned}
$$

where we have used the integral identity [7, Eq. (4.352.1)]. Further noticing that the dominant term in the first item of (5) is the one for $k=2$, we can obtain the desired result after some simple algebraic manipulations.

As we can observe, there is a scaling of 2 multiplying the first term in (7), which indicates that the sum rate of MIMO Nakagami- $m$ channels with MMSE receivers still scales linearly with the minimum number of antennas in the high SNR regime. This is in line with the associated results on optimal ML receivers [2]. Figure 1 examines the tightness of the proposed upper bound given in Proposition 1. For our simulation purposes, we have set $m=2$ and $N_{t}=2$. As we can readily observe, the proposed upper bound is in general quite tight, especially for low to medium SNR values. As anticipated, the tightness of the upper bound improves when $N_{r}$ becomes larger and in the limit of large $N_{r}$, the bound becomes exact. Furthermore, it is evident that the sum rate scales linearly with $N_{t}$, and increasing $N_{r}$ provides a logarithmic array gain.

\section{B. Low SNR Analysis}

We now look into the low SNR regime, and provide a detailed sum rate characterization of MIMO Nakagami- $m$ 


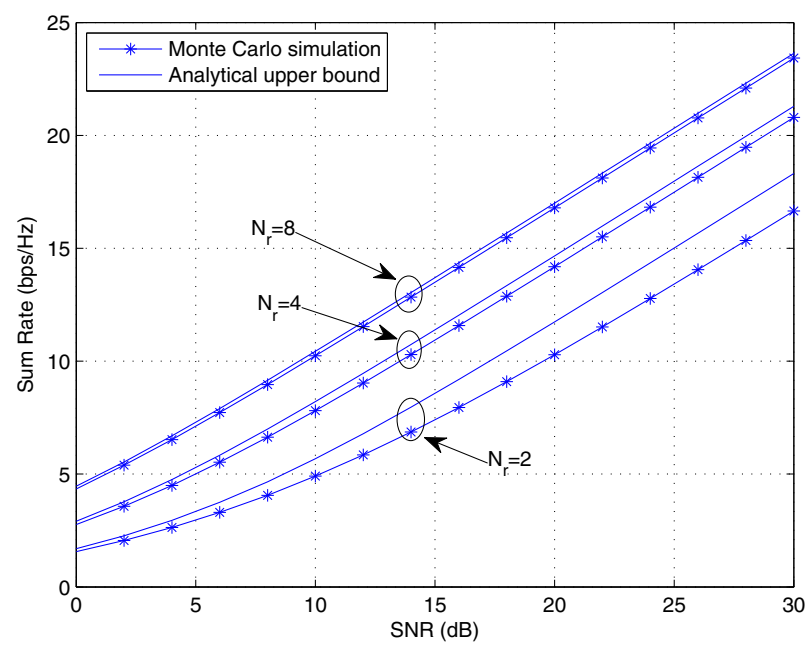

Fig. 1. Ergodic sum rate of MIMO Nakagami- $m$ fading channels with MMSE receivers when $N_{t}=2$ and $m=2$ : Monte Carlo simulations vs. Analytical upper bound.

fading channels with MMSE receivers. We first recall that, as it was explicitly demonstrated in [11], the low SNR capacity of MIMO systems can be more accurately captured via the normalized transmit energy per information bit $E_{b} / N_{0}$ rather than via the per-symbol SNR. This capacity representation, also adopted in [12] among others, reads as

$$
\mathcal{R}\left(\frac{E_{b}}{N_{0}}\right) \approx \mathcal{S}_{0} \log _{2}\left(\frac{\frac{E_{b}}{N_{0}}}{\frac{E_{b}}{N_{0} \text { min }}}\right)
$$

where $\frac{E_{b}}{N_{0}}$ and $\mathcal{S}_{0}$ are the two key parameters dictating the low SNR behavior, corresponding to the minimum normalized energy per information bit required to convey any positive rate reliably and the wideband slope, respectively. Following [11], these two figures of merit are defined as

$$
{\frac{E_{b}}{N_{0} \text { min }}}=\frac{1}{\dot{\mathcal{R}}(0)}, \quad \mathcal{S}_{0}=-2 \ln 2 \frac{(\dot{\mathcal{R}}(0))^{2}}{\ddot{\mathcal{R}}(0)},
$$

where $\dot{\mathcal{R}}(\cdot), \ddot{\mathcal{R}}(\cdot)$ denote the first and second-order derivatives of the sum rate (4) over the SNR $\rho$, respectively. Before presenting our main results, we recall the following expressions that hold for MIMO Nakagami- $m$ fading channels with optimal receivers:

Lemma 1 ([13]): For $N_{r} \times N_{t}$ MIMO Nakagami- $m$ fading channels with optimal receivers, the minimum energy per information bit to reliably convey any positive rate and the wideband slope are respectively given by

$$
\begin{aligned}
{\frac{E_{b}}{N_{0}{ }_{\text {min }}^{\text {opt }}}}^{\text {opt }} & =\frac{\ln 2}{N_{r} \Omega} \\
S_{0}^{\text {opt }} & =\frac{2 N_{r} N_{t}}{N_{r}+N_{t}-1+1 / m} .
\end{aligned}
$$

With these definitions in hand, we can now present the following novel result:

Proposition 2: For $N_{r} \times N_{t}$ MIMO Nakagami-m fading channels with MMSE receivers, the minimum energy per information bit to reliably convey any positive rate and the wideband slope are respectively given by

$$
\begin{aligned}
{\frac{E_{b}}{N_{0}}}_{\text {min }}^{\text {mmse }} & =\frac{\ln 2}{N_{r} \Omega} \\
S_{0}^{\text {mmse }} & =\frac{2 N_{r} N_{t}}{N_{r}+2 N_{t}-2+1 / m} .
\end{aligned}
$$

Proof: See Appendix A.

Interestingly, the minimum required $\frac{E_{b}}{N_{0}}$ is independent of the Nakagami- $m$ factor and number of transmit antennas. The impact of the former parameter can be assessed only via the wideband slope, which is an increasing function of $m$. Moreover, it is easily seen that $m$ has a noticeable impact on $S_{0}^{\text {mmse }}$ for small number of antennas, while its impact is significantly reduced for higher number of antennas. In fact, we can trivially upper $(m \rightarrow \infty)$ and lower $(m=0.5)$ bound $S_{0}^{\mathrm{mmse}}$ as follows:

$$
\frac{2 N_{r} N_{t}}{N_{r}+N_{t}-2} \leq S_{0}^{\text {mmse }} \leq \frac{2 N_{r} N_{t}}{N_{r}+2 N_{t}} .
$$

We note that for Rayleigh fading conditions (i.e. $m=1$ ), (13)(14) respectively coincide with [5, Eq. (33)] and [5, Eq. (52)]. Comparing now (11) with (13), we can easily infer that the MMSE receiver is optimal in terms of $\frac{E_{b}}{N_{0}}$ min . On the other hand, the sub-optimality of the MMSE receiver is reflected via a reduced $S_{0}$. This can be clearly observed in Fig. 2, which illustrates the low SNR sum rate of optimal and MMSE receivers for different MIMO configurations. Moreover, we see that the relative difference between the sum rate curves increases for larger MIMO configurations. This implies that when the number of transmit antennas $N_{t}$ increases, MMSE receivers perform poorly due to the increased number of interfering streams. In particular, we have that

$$
\frac{S_{0}^{\mathrm{opt}}}{S_{0}^{\text {mmse }}}=1+\frac{N_{t}-1}{N_{r}+N_{t}-1+1 / m} .
$$

The above ratio is decreasing in $N_{r}$ and increasing in $N_{t}$, thereby demonstrating the improved interference cancellation capability of MMSE receivers for a high number of receive antennas $N_{r}$. In addition, for fixed $N_{r}$ and $m$, (16) can be bounded as follows:

$$
1 \leq \frac{S_{0}^{\mathrm{opt}}}{S_{0}^{\text {mmse }}}<2 .
$$

The lower bound in (17) is attained only when $N_{t}=1$. This is anticipated, since MMSE receivers become optimal for a single transmit antenna [8]. The upper bound in (17) is obtained when $N_{t} \rightarrow \infty$, which indicates the inherently poor performance of the MMSE receiver when the number of transmit antennas exceeds the number of receive antennas. Note that similar conclusions were also drawn in [5]. 


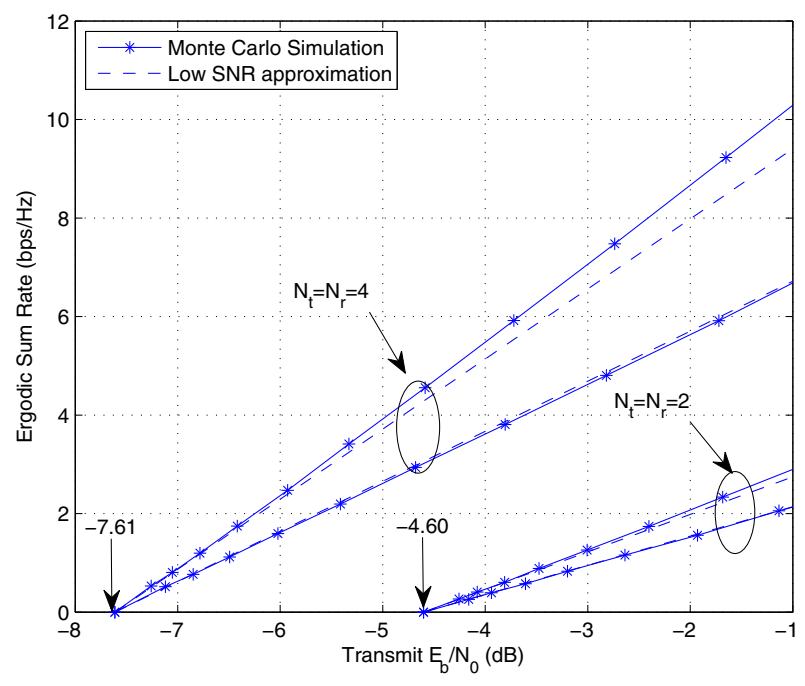

Fig. 2. Comparison of the low SNR sum rate of MIMO Nakagami- $m$ fading channels with optimal and MMSE receivers.

\section{CONCLUSiON}

This paper has investigated the achievable sum rate of MIMO Nakagami- $m$ fading channels with linear MMSE receivers. New, closed-form upper bounds were obtained for the achievable sum rate of dual transmit antenna configurations. Moreover, exact expressions for the minimum energy per information bit to reliably convey any positive rate and wideband slope were deduced. The presented results explicitly indicate that the sum rate loss due to the MMSE receivers is caused by a reduction in the wideband slope.

\section{APPENDIX A}

\section{ProOF OF PROPOSITION 2}

The proof starts by following the generic methodology of [5]. On this basis, recalling the following key matrix property

$$
\left[\mathbf{A}^{-1}\right]_{i, i}=\frac{\operatorname{det}\left(\mathbf{A}_{i i}\right)}{\operatorname{det}(\mathbf{A})}
$$

where $\mathbf{A}_{i i}$ is the $(i, i)$-th minor of matrix $\mathbf{A}$, we can re-express (4) as follows

$$
\begin{aligned}
& \mathcal{R}^{\text {mmse }}(\rho)=N_{t} \mathrm{E}_{\mathbf{H}}\left\{\log _{2} \operatorname{det}\left(\mathbf{I}_{N_{t}}+\frac{\rho}{N_{t}} \mathbf{H}^{\dagger} \mathbf{H}\right)\right\} \\
& -\sum_{i=1}^{N_{t}} \mathrm{E}_{\mathbf{H}_{i}}\left\{\log _{2} \operatorname{det}\left(\mathbf{I}_{N_{t}-1}+\frac{\rho}{N_{t}} \mathbf{H}_{i}^{\dagger} \mathbf{H}_{i}\right)\right\}
\end{aligned}
$$

where $\mathbf{H}_{i}$ is $\mathbf{H}$ with the $i$-th column removed. Starting from (19), we apply the following properties for the derivatives of determinants

$$
\begin{gathered}
\left.\frac{d}{d x} \ln \operatorname{det}(\mathbf{I}+x \mathbf{A})\right|_{x=0}=\operatorname{tr}(\mathbf{A}) \\
\left.\frac{d^{2}}{d x^{2}} \ln \operatorname{det}(\mathbf{I}+x \mathbf{A})\right|_{x=0}=-\operatorname{tr}\left(\mathbf{A}^{2}\right)
\end{gathered}
$$

which give

$$
\begin{aligned}
\dot{\mathcal{R}}^{\text {mmse }}(0) & =\frac{1}{\ln 2}\left(\mathrm{E}_{\mathbf{H}}\left\{\operatorname{tr}\left(\mathbf{H}^{H} \mathbf{H}\right)\right\}\right. \\
& \left.-\frac{1}{N_{t}} \sum_{i=1}^{N_{t}} \mathrm{E}_{\mathbf{H}_{i}}\left\{\operatorname{tr}\left(\mathbf{H}_{i}^{H} \mathbf{H}_{i}\right)\right\}\right)
\end{aligned}
$$

and

$$
\begin{aligned}
\ddot{\mathcal{R}}^{\mathrm{mmse}}(0) & =-\frac{1}{N_{t} \ln 2} \mathrm{E}_{\mathbf{H}}\left\{\operatorname{tr}\left(\left[\mathbf{H}^{H} \mathbf{H}\right]^{2}\right)\right\} \\
& +\frac{1}{N_{t}^{2} \ln 2} \sum_{i=1}^{N_{t}} \mathrm{E}_{\mathbf{H}_{i}}\left\{\operatorname{tr}\left(\left[\mathbf{H}_{i}^{H} \mathbf{H}_{i}\right]^{2}\right)\right\} .
\end{aligned}
$$

The desired results can be obtained after evaluating the involved expectations in (22), (23) with the aid of the results from [13, Theorem 2], and simplifying.

\section{ACKNOWLEDGEMENTS}

The work of M. Matthaiou has been supported in part by the Swedish Governmental Agency for Innovation Systems (VINNOVA) within the VINN Excellence Center Chase.

\section{REFERENCES}

[1] I. E. Telatar, "Capacity of multi-antenna Gaussian channels," Europ. Trans. Telecommun., vol. 10, no. 6, pp. 585-595, Nov./Dec. 1999.

[2] A. Grant, "Rayleigh fading multi-antenna channels," EURASIP J. Appl. Signal Process., vol. 2002, no. 3, pp. 316-329, Mar. 2002.

[3] H. Shin and J. H. Lee, "Capacity of multi-antenna fading channels: Spatial fading correlation, double scattering, and keyhole," IEEE Trans. Inf. Theory, vol. 49, no. 10, pp. 2636-2647, Oct. 2003.

[4] C. Zhong, S. Jin, K.-K. Wong, and M. R. McKay, "Ergodic mutual information analysis for multi-keyhole MIMO channels," IEEE Trans. Wireless Commun., vol. 10, no. 6, pp. 1754-1763, June 2011.

[5] M. R. McKay, I. B. Collings, and A. M. Tulino, "Achievable sum rate of MIMO MMSE receivers: A general analytic framework," IEEE Trans. Inf. Theory, vol. 56, no. 1, pp 396-410, Jan. 2010.

[6] M. Nakagami, "The $m$-distribution-A general formula of intensity distribution of rapid fading," in Statistical Methods in Radio Wave Propagation, W. C. Hoffman Ed., pp. 3-36, Oxford, U.K.: Pergamon, 1960.

[7] I. S. Gradshteyn and I. M. Ryzhik, Table of Integrals, Series, and Products, Seventh Ed. Academic Press, San Diego, 2007.

[8] D. Tse and P. Viswanath, Fundamentals of Wireless Communication, Cambridge University Press, 2005.

[9] C. Zhong, M. Matthaiou, A. Huang, and Z. Zhang, "On the sum rate of MIMO Nakagami- $m$ fading channels with linear receivers," submitted to IEEE Trans. Wireless Commun., Nov. 2011.

[10] C. Zhong, K.-K. Wong, and S. Jin, "Capacity bounds for MIMO Nakagami-m fading channels," IEEE Trans. Signal Process., vol. 57, no. 9, pp. 3613-3623, Sept. 2009.

[11] S. Verdú, "Spectral efficiency in the wideband regime," IEEE Trans. Inf. Theory, vol. 48, no. 6, pp. 1319-1343, June 2001.

[12] A. Lozano, A. M. Tulino, and S. Verdú, "Multiple-antenna capacity in the low-power regime," IEEE Trans. Inf. Theory, vol. 49, no. 10, pp. $2527-2544$, Oct. 2003.

[13] C. Zhong, S. Jin, T. Ratnarajah, and K.-K. Wong, "On the capacity of non-uniform phase MIMO Nakagami- $m$ fading channels," IEEE Commun. Lett., vol. 14, no. 6, pp. 536-538, June 2010. 